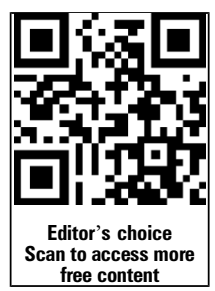

- Additional material is published online only. To view please visit the journal online (http://dx.doi.org/10.1136/ bjsports-2014-094527).

${ }^{1}$ Sports Medicine, Australian Institute of Sport, Bruce, Australia

${ }^{2}$ Rowing Australia, Melbourne, Australia

${ }^{3}$ Sports Medicine, Australian Paralympic Team, Sydney, Australia

${ }^{4}$ Sports Medicine, Australian Institute of Sport, Canberra, Australia

\section{Correspondence to} Dr Stacey Compton, Australian Institute of Sport, Leverrier Crescent, Bruce, ACT 2167, Australia; stacey.compton@ uqconnect.edu.au

Accepted 18 March 2015 Published Online First 13 April 2015
CrossMark

$$
\begin{aligned}
& \text { To cite: Compton S, } \\
& \text { Trease L, Cunningham C, } \\
& \text { et al. Br J Sports Med }
\end{aligned}
$$$$
\text { 2015;49:1236-1240. }
$$

\title{
Australian Institute of Sport and the Australian Paralympic Committee position statement: urinary tract infection in spinal cord injured athletes
}

\author{
Stacey Compton, ${ }^{1}$ Larissa Trease, ${ }^{2}$ Corey Cunningham, ${ }^{3}$ David Hughes ${ }^{4}$
}

\section{ABSTRACT}

Patients with spinal cord injuries are at increased risk of developing symptomatic urinary tract infections. Current evidence-based knowledge regarding prevention and treatment of urinary tract infection in the spinal cord injured population is limited. There are currently no urinary tract infection prevention and management guidelines specifically targeted towards elite spinal cord injured athletes. This position statement represents a set of recommendations intended to provide clinical guidelines for sport and exercise medicine physicians and other healthcare providers for the prevention and treatment of urinary tract infection in spinal cord injured athletes. It has been endorsed by the Australian Institute of Sport (AIS) and the Australian Paralympic Committee (APC).

\section{INTRODUCTION}

Patients with a spinal cord injury (SCI) are at increased risk of developing symptomatic urinary tract infections (UTIs) ${ }^{1-4}$ as a result of impaired storage and voiding function. ${ }^{5} 6$ The evidence base is limited, making it difficult to recommend evidence-based guidelines for the prevention and treatment of UTI in the SCI population. ${ }^{6} 7$ A survey of UTI treatment for patients with SCI in centres specialising in the urological treatment in Germany, Switzerland and Austria reported that treatment is not well standardised. ${ }^{6}$ The evidence base specific to Paralympic athletes is also limited. ${ }^{8}$ Additionally, there are no UTI guidelines specifically targeted towards elite athletes.

A study of 61 SCI athletes who used intermittent catheterisation reported that athletes from developed nations experienced an average of 1.6 UTIs per year. Athletes from developing nations experienced on average 3.5 UTIs per year. ${ }^{9}$ Furthermore, Derman et $a l^{10}$ reported a $7.6 \%$ frequency of genitourinary illness in Paralympic athletes during the competition period of the London 2012 Paralympic games. This is higher than that reported in similar studies of able-bodied athletes. ${ }^{10}$ UTIs during competition may prevent athletes from competing. ${ }^{9}$

This position statement represents a set of recommendations intended to provide clinical guidelines for sport and exercise medicine (SEM) physicians and other healthcare providers for the prevention and treatment of UTI in SCI athletes. The recommendations were developed based on a review of the current literature regarding UTI in patients with SCI (boxes 1 to 4). This position statement has been endorsed by the Australian
Institute of Sport (AIS) and the Australian Paralympic committee (APC).

\section{ASYMPTOMATIC BACTERIURIA AND DIPSTICK TESTING \\ Asymptomatic bacteriuria}

Asymptomatic bacteriuria is a common finding in patients with SCI. ${ }^{1}{ }^{11}$ Prevalence is over 50\%, regardless of whether voiding is managed by intermittent catheterisation or by sphincterotomy and condom drainage. Prevalence approaches 100\% in patients utilising long-term indwelling catheterisation. $^{12-15}$ The Infectious Diseases Society of America define asymptomatic bacteriuria as $\geq 10^{5} \mathrm{cfu} / \mathrm{mL}$ in two consecutive urine samples collected in a manner to minimise contamination. ${ }^{16}$ The European Association of Urology recommend that asymptomatic bacteriuria in patients with SCI should not be treated. ${ }^{17}$ This is supported by several other guidelines and authors ${ }^{3} 4 \begin{array}{lllll}4 & 16 & 18 & 19\end{array}$ as it has not been shown to be effective, and can $\begin{array}{llllll}\text { create antimicrobial resistance. }{ }^{4} & 7 & 11 & 20 & 21\end{array}$ Additionally, bacteriuria may indicate poor hydration or infrequent catheterisation, and may be addressed by changing hydration and voiding/ bladder management routines. ${ }^{13}$

\section{Dipstick testing}

Several guidelines and authors state that routine urinary dipstick or microscopy, culture and sensitivity $(\mathrm{m} / \mathrm{c} / \mathrm{s})$ testing is not recommended in patients with SCI. ${ }^{7} 161719$ Pyuria is common in patients with catheters, and has no predictive value in differentiating symptomatic UTI from asymptomatic bacteriuria. ${ }^{322-24}$ Additionally, pyuria in association with asymptomatic bacteriuria is not an indication for antibiotic treatment. ${ }^{13} 1316$

\section{DIAGNOSIS OF UTI}

\section{Signs and symptoms of UTI}

UTI in patients with SCI is defined by both presence of physical symptoms and high amounts of bacteriuria. $^{3} 4{ }^{4} 13{ }^{24}$ In the asymptomatic patient, urine odour, cloudiness, and/or the presence of pyuria are not sufficient to indicate infection. ${ }^{1} 31323$

There are no standard criteria for signs or symptoms constituting symptomatic UTI in patients with SCI. ${ }^{6}{ }^{18}$ In catheterised patients, the common occurrence of fever and bacteriuria and the variable presence of a broad range of other associated clinical manifestations make the diagnosis of symptomatic UTI difficult. ${ }^{3}$

The following symptoms have been suggested as potential indicators of a UTI in patients with SCI: 
Box 1 Recommendations relating to asymptomatic bacteriuria and dipstick testing in spinal cord injured athletes

1. Routine dipstick testing should not be used

2. Asymptomatic bacteriuria in spinal cord injured athletes should not be treated

Box 2 Recommendations relating to diagnosis and treatment of urinary tract infection (UTI) in spinal cord injured athletes

1. Urine should be sent for microscopy, culture and sensitivity $(\mathrm{m} / \mathrm{c} / \mathrm{s})$ in spinal cord injured athletes presenting with symptoms potentially indicating UTI

2. Indwelling catheters should be changed prior to collection of urine

3. Urine should be tested prior to initiation of antibiotics, and antibiotic choice re-evaluated once the urine $\mathrm{m} / \mathrm{c} / \mathrm{s}$ results are available

- Acute or worsening fever

- Rigours

- Altered mental state

- Lethargy or malaise (with no other identifiable cause)

- Acute haematuria

- Dysuria

- Flank or pelvic discomfort

- Development of or increase of urinary incontinence

- Increased frequency of catheterisation/voiding

- Increased spasticity

- Autonomic dysreflexia

- Sense of unease. ${ }^{24}$

\section{Testing}

Urine should be sent for $\mathrm{m} / \mathrm{c} / \mathrm{s}$ where one or more of the aforementioned symptoms are present. ${ }^{3} 1324$ As regular catheterisation is associated with bacteriuria, reliance on dipstick testing for nitrite and leucocyte esterase only can lead to false-positive diagnoses of UTI. Evaluation with urine culture should be used to ensure proper diagnosis and treatment. ${ }^{25}$ Long-term indwelling catheters should be changed prior to collection of urine as this improves the accuracy of culture results. ${ }^{3} 18$

When travelling with teams, access to urine $\mathrm{m} / \mathrm{c} / \mathrm{s}$ may not always be available. The travelling team physician may wish to consider using a urine dipstick in these circumstances to augment their clinical findings. However, urine $\mathrm{m} / \mathrm{c} / \mathrm{s}$ is at all times preferable for diagnosing UTI. If there is suspicion regarding UTI, it is recommended that the travelling team physician attempt to gain access to this investigation.

There are no standard criteria for levels of bacteriuria constituting symptomatic UTI in patients with SCI. ${ }^{18} 26$ The following has been suggested as quantitative urine culture criteria for the diagnosis of bacteriuria in individuals with SCI:

- Catheter specimens from SCI on intermittent catheterisation: $\geq 10^{2} \mathrm{cfu} / \mathrm{mL}$

- Clean catch specimens from catheter-free males using condom collection devices: $\geq 10^{4} \mathrm{cfu} / \mathrm{mL}$

- Specimens from indwelling catheters or suprapubic aspirates: any detectable concentration. ${ }^{24}$
MANAGEMENT OF UTI

\section{Antibiotic treatment}

Antibiotic therapy is only indicated for symptomatic UTI. ${ }^{7} 17$ Empirical therapy should be started based on suspected causative organisms' antibiotic sensitivities, and then replaced by a therapy adjusted for the specific organism(s) identified in the urine culture. ${ }^{17} 24$

The majority of uncomplicated UTIs in Australia can be treated with trimethoprim, cephalexin or amoxicillin with clavulanate. $^{27}$ The European Association of Urology state that there is no superiority of one agent or class of antimicrobials for treatment of UTI in patients with SCI. ${ }^{17}$ In complicated UTIs (including patients with neurogenic bladder) therapy should be guided by culture results. ${ }^{27}$ Patients with SCI with previous UTIs could be started on the antibiotic the organism was previously sensitive to, and this then reviewed once culture results have become available. Where there are medical comorbidities, such as an athlete with one kidney, specialist advice should be sought early to guide treatment. Additionally, in some parts of the world (eg, South East Asia), there is high antibiotic resistance to Escherichia coli, which is an organism which commonly causes UTI. ${ }^{28}$ Therefore, a low threshold for specialist review should be considered for UTI in SCI athletes during or after travel to these countries.

The optimal duration of antibiotic treatment has not been established. ${ }^{18}$ Therapy for $7-10$ days is the most commonly accepted protocol. ${ }^{17}$ The Infectious Diseases Society of America guidelines recommend a 7-day course of antibiotics for patients with symptomatic catheter-associated UTI who have prompt resolution of symptoms, or 10-14 days if there is a delayed response. $^{29}$

\section{UTI PREVENTION}

\section{Cranberries}

Cranberries (particularly juice) are widely recommended for use to prevent and treat UTIs. ${ }^{2}$ Evidence of the efficacy of cranberries in prevention of UTIs is however inconsistent. ${ }^{13}$ A recent Cochrane review assessing the effectiveness of cranberry products in preventing UTIs, reported that cranberry products did not significantly reduce symptomatic UTI recurrence in patients with neuropathic bladder or SCI. ${ }^{2}$

\section{Methenamine salts}

A Cochrane review investigating the use of methenamine hippurate in the prevention of UTI concluded that it may be useful in patients without renal abnormalities, particularly if used for short-term prophylaxis. However, in patients with renal tract abnormalities or neuropathic bladder, methenamine salts do not appear to be effective in the prevention of UTI. ${ }^{30}$

\section{Other acidification/alkalinisation agents}

A systematic review reported that ammonium chloride, sodium bicarbonate, acetazolamide, and ascorbic acid are not useful in preventing UTI in patients with neurogenic bladder. ${ }^{7}$

\section{Catheters}

Intermittent catheterisation is the gold standard for treating voiding disorders associated with neurogenic bladder ${ }^{13}{ }^{31}$ and is associated with a lower incidence of UTI and asymptomatic bacteriuria. $^{347}$ A Cochrane review regarding intermittent catheterisation concluded that there is a lack of reliable evidence that any specific catheter type (coated or uncoated), method (single or multiple use), technique (sterile or clean), person (self 
or other) or strategy is better than any other for all clinical settings. ${ }^{32}$ However, a recent meta-analysis supported the use of hydrophilic catheters in individuals with SCI, as intermittent catheterisation with hydrophilic catheters was associated with less UTIs and haematuria. ${ }^{33}$

\section{Bladder management}

A bladder management programme is necessary to assist bladder emptying in individuals with SCI. ${ }^{5}$ Such a programme should enable bladder emptying on a frequent schedule while allowing patients to remain free from urinary leakage while storing urine at low bladder pressures. ${ }^{31}$ There is a risk of UTI if the bladder does not empty completely. ${ }^{5}$ Poor bladder management technique includes inadequately cleaning catheters, inadequate hand washing or perineal hygiene, excessively short or long intervals between catheterisation, or contaminating the catheter during insertion. ${ }^{13}$ The European Association of Urology recommend anticholinergic medications and clean intermittent catheterisation as first-line management for individuals with SCI who are unable to empty their bladder. ${ }^{17}$

Frequent bladder emptying by catheterisation is an important factor in preventing UTI. $^{7}$ The conservative methods for bladder management include indwelling catheterisation, intermittent catheterisation, and condom catheterisation. Intermittent catheterisation is the preferred method, due to reduced incidence of urological complications compared with other methods. ${ }^{4}$ Intermittent catheterisation should be carried out on a regular basis, usually every 4-6 h, to keep the amount of urine obtained to less than $500 \mathrm{~mL}$ per collection. ${ }^{34}$ More frequent catheterisation increase risk of cross-infection, while less frequent result in high bladder storage volumes. ${ }^{3}$

Hand hygiene is an important preventative measure. ${ }^{5}$ Individuals with SCI should be taught hand hygiene and clean catheterisation technique to minimise the risk of UTI. ${ }^{34}$ A Cochrane review reported that there was a lack of reliable evidence regarding sterile technique versus clean for reducing UTI in patients with SCI. ${ }^{32}$ It is therefore recommended that clean catheterisation technique should be taught. Sterile catheterisation should be considered for individuals with recurrent symptomatic infections occurring with clean catheterisation technique. $^{34}$

Hand hygiene should include teaching to clean hands with either antibacterial soap or alcohol-based cleaners prior to and after catheter insertion, manipulating the catheter or changing of drainage systems. Additionally, daily catheter hygiene should be performed, including cleaning of the perineal region and proximal catheter with soap and water for patients with an indwelling catheter. ${ }^{5}$ Patients using intermittent catheterisation should use catheters for single use only, as per manufacturer guidelines. A study of $61 \mathrm{SCI}$ athletes who used intermittent catheterisation reported that athletes who reused catheters experienced significantly more frequent UTI. Athletes from developing nations were significantly more likely to reuse catheters, and exhibited two times more UTIs per year than athletes from developed nations. The authors suggested that catheter reuse may be due to a lack of resources or a lack of education. ${ }^{9}$ All athletes utilising intermittent catheterisation should be educated regarding catheter hygiene, including that catheters are for single use only.

Adequate hydration may also decrease the risk of developing UTI. ${ }^{13} 24 \quad 35 \quad 36$ Unless contraindicated, recommended fluid intake should be $2-3 \mathrm{~L}$ per day. ${ }^{24}$ Variability in exercise intensity or climatic conditions should be taken into account when
Box 3 Recommendations relating to prevention of urinary tract infection (UTI) in spinal cord injured athletes

1. Cranberries products, methenamine salts, and acidification/ alkalinisation products cannot be recommended for UTI prevention

2. Antibiotic prophylaxis should not be routinely prescribed to prevent symptomatic UTI

3. Spinal cord injured (SCI) athletes with recurrent UTIs should be medically reviewed in relation to whether antibiotic prophylaxis would be appropriate

4. Travelling athletes who choose to dehydrate should be reviewed by their general practitioner or sport and exercise medicine physician to discuss whether antibiotic prophylaxis is appropriate

5. Education should be provided to all $\mathrm{SCl}$ athletes regarding UTI. Education should include several sessions, and verbal and written information should be provided. This information may include, but not be limited to:

- Signs and symptoms of UTI

- Prevention of UTI

- Treatment of UTI

- Hand hygiene

- Perineal hygiene

- Frequent bladder emptying

- Use of catheters

- Adequate hydration

Box 4 Additional recommendations based on current practice

Alkalise the urine during acute urinary tract infection for symptomatic relief if required

considering fluid intake requirements. During acute UTIs, bladder pressure should be lowered by increasing the frequency of bladder emptying, and by consideration of anticholinergic drugs. $^{7}$

\section{Antibiotic prophylaxis}

The benefits of long-term antibiotic prophylaxis remains unproven. ${ }^{18}$ The Scottish Intercollegiate Guidelines Network recommends that antibiotic prophylaxis should not be routinely prescribed to prevent symptomatic UTI in patients with catheters. It is suggested that antibiotic prophylaxis should be considered for patients with whom frequency or severity of UTI is chronically impinging on function and well-being. ${ }^{3}$ Frequent UTI is defined in patients with SCI as three or more UTIs per year where both a positive urine culture and infection symptoms are present. ${ }^{13}$ Additionally it is recommended that antibiotic prophylaxis should only be used for people with a history of catheter-associated UTI following catheter change. ${ }^{3}$

A pilot study into prevention of UTI in patients with SCI however reported a significant decrease in UTI and hospitalisation using a weekly oral cyclic antibiotic (WOCA) regime over a 2 -year period. ${ }^{37}$ The WOCA regime consisted of alternate antibiotic administration once per week over a 2 -year period. The antibiotics used included: amoxicillin $3000 \mathrm{mg}$, cefixime 
$400 \mathrm{mg}$, fosfomycin trometamol $6000 \mathrm{mg}$, nitrofurantoin $300 \mathrm{mg}$, and trimethoprim/sulfamethoxazole $320-1600 \mathrm{mg}$. The antibiotics were specifically chosen for each patient according to the results of urine culture.

Elite athletes are required to travel for training, and to compete in interstate and international competition. SCI athletes often choose to dehydrate in order to minimise the number of times catheterisation is needed. These athletes are at increased risk of UTI, and it is recommended they see their general practitioner or SEM physician to discuss antibiotics for prevention of UTI during travel. If antibiotic prophylaxis is instituted for these athletes during travel, this should be based on previous causative organisms' antibiotic sensitivity. It is however recommended that techniques to ensure adequate hydration during travelling should be discussed prior to considering antibiotic prophylaxis. Additionally, it is recommended that hand luggage includes adequate numbers of catheterisation equipment, and that seating preferences are considered to allow adequate access to toilet facilities.

\section{Education}

Mays et $a l^{38}$ reported that educational programmes may be beneficial in reducing UTIs in individuals with SCI. However, there is no evidence suggesting the efficacy of one education approach over another in reducing UTIs. A prospective cohort study into the effect of nursing education on UTI recurrence in patients with spinal cord injuries found that $65 \%$ of patients responded to education. Of this cohort however, 73\% required multiple sessions to achieve a decline in the number of UTIs below threshold. Education included catheterisation techniques, cleaning of the perineum, use of examination gloves and cleaning of catheters. ${ }^{39}$

\section{CURRENT PRACTICE}

The following is recommended in relation to UTI prevention in individuals with SCI by spinal units in Australia:

- Encourage adequate hydration (2-2.5 L/day unless other contraindications)

- Educate regarding hand washing and catheter hygiene and technique

- Education regarding signs and symptoms of UTI

- Recommend vitamin C $1 \mathrm{~g}$ two times per day and methenamine hippurate $1 \mathrm{~g}$ two times per day for UTI prevention (NB: this is not supported in the literature). Cranberries may be utilised for UTI prevention if patient preference

- Twelve monthly reviews with urologist

The following is recommended in relation to UTI management in individuals with SCI by spinal units in Australia:

- No need to treat asymptomatic bacteriuria

- Change catheter prior to taking urine sample

- Antibiotic choice based on urine sensitivities

- Alkalise the urine with Ural if required for symptomatic relief

- Ensure adequate hydration (2-2.5 L/ day unless other contraindications).

There is common use of vitamin C, methenamine hippurate and cranberries for UTI prevention in patients with SCI. The evidence base currently does not support this, practice. There are, however, no documented significant adverse effects.

\section{RECOMMENDATIONS}

A summary of recommendations for UTI prevention and management in SCI athletes has been provided (box 5). Please
Box 5 Summary of recommendations for urinary tract

infection (UTI) prevention and management in spinal cord injured athletes

1. Routine urine dipsticks or cultures are not required. Bacteriuria is a common finding in asymptomatic, catheterised athletes

2. Treatment should only be considered in the presence of signs and symptoms. Urine should be sent for microscopy, culture and sensitivity $(\mathrm{m} / \mathrm{c} / \mathrm{s})$ if UTI is clinically suspected

3. Indwelling catheters should be changed prior to collection of urine for testing

4. Urine should be tested prior to initiation of antibiotics, and antibiotic choice re-evaluated once $\mathrm{m} / \mathrm{c} / \mathrm{s}$ results are available

5. Alkalise the urine during acute UTI for symptomatic relief, if required

6. Cranberries, methenamine salts, acidifying or alkalinising agent cannot be recommended for the prevention of UTI based on the current literature

7. Antibiotic prophylaxis should not be routinely prescribed to prevent symptomatic UTI

8. Spinal cord injured ( $\mathrm{SCl}$ ) athletes with recurrent UTIs, or those who choose to dehydrate for travel should be medically reviewed in relation to whether antibiotic prophylaxis would be appropriate

9. All $\mathrm{SCl}$ athletes should receive education regarding prevention, signs and symptoms, and treatment of UTI. This may include, but not be limited to:

- Signs and symptoms of UTI

- Prevention of UTI

- Treatment of UTI

- Hand hygiene

- Perineal hygiene

- Frequent bladder emptying

- Use of catheters

- Adequate hydration

Education should include several sessions, and verbal and written information should be provided

refer to online supplementary Appendix A for a flow chart designed to be used by travelling teams for the prevention and management of UTI. Online supplementary Appendix B contains a patient handout relating to UTI prevention and management.

\section{What are the new findings?}

- There are no urinary tract infection guidelines specifically targeted towards elite spinal cord injured athletes.

- This position statement summarises the current evidence for urinary tract infection prevention and management in spinal cord injured patients.

- A set of recommendations for use in spinal cord injured athletes and a flow chart designed for use during travel are provided. 


\section{How might it impact on clinical practice in the near future?}

- This position statement represents a set of recommendations intended to provide clinical guidelines for the prevention and treatment of urinary tract infection in spinal cord injured athletes.

- It is intended that this will aid sport and exercise medicine physicians and other healthcare providers in evidence-based healthcare provision for spinal cord injured athletes.

- This position statement has been endorsed by the Australian Institute of Sport (AIS) and the Australian Paralympic Committee (APC).

Acknowledgements The authors wish to thank the following individuals for their valuable review of this document: Dr Greg Lovell, Australian Institute of Sport; and Dr Nicole Vlahovich, Australian Institute of Sport. The authors also wish to thank Samantha Waight, Spinal Urology Clinical Nurse Consultant Victorian Spinal Cord Service (Austin Health) for discussions regarding current practice in spinal cord units.

Contributors SC was responsible for the planning, conduct and reporting of the information within the manuscript. LT was involved in the planning and review of the manuscript. CC was involved in the planning and review of the manuscript. DH was involved in the planning and review of the manuscript. DH has the authority to endorse this position statement on behalf of the AIS Department of Sports Medicine.

Competing interests None.

Provenance and peer review Not commissioned; externally peer reviewed.

\section{REFERENCES}

1 Colgan R, Nicolle LE, McGlone A, et al. Asymptomatic bacteriuria in adults. Am Fam Physician 2006;74:985-90.

2 Jepson RG, Williams G, Craig JC. Cranberries for preventing urinary tract infections. Cochrane Database Syst Rev 2012;10:Cd001321.

3 SIGN. Management of suspected bacterial urinary tract infection in adults Edinburgh. SIGN, 2012 [cited 2012]. http://www.sign.ac.uk

4 Wolfe DL, Legassic M, Mclntyre A, et al. Bladder health and function following spinal cord injury. Vancouver: Spinal Cord Injury Rehabilitation Evidence, 2012. 4.0. http://www.scireproject.com/rehabilitation-evidence/bladder-management

5 Eves FJ, Rivera N. Prevention of urinary tract infections in persons with spinal cord injury in home health care. Home Healthc Nurse 2010;28:230-41.

6 Pannek J. Treatment of urinary tract infection in persons with spinal cord injury: guidelines, evidence, and clinical practice. A questionnaire-based survey and review of the literature. J Spinal Cord Med 2011;34:11-15.

7 Everaert K, Lumen N, Kerckhaert W, et al. Urinary tract infections in spinal cord injury: prevention and treatment guidelines. Acta Clin Belg 2009;64:335-40.

8 Thompson WR, Vanlandewijck YC. Science and the Paralympic movement. $\mathrm{Br} J$ Sports Med 2013;47:811.

9 Krassioukov A, Cragg JJ, West C, et al. The good, the bad and the ugly of catheterization practices among elite athletes with spinal cord injury: a global perspective. Spinal Cord 2015;53:78-82.

10 Derman W, Schwellnus $M$, Jordaan E, et al. Illness and injury in athletes during the competition period at the London 2012 Paralympic Games: development and implementation of a web-based surveillance system (WEB-IISS) for team medical staff. Br J Sports Med 2013;47:420-5.

11 Hinkel A, Finke W, Botel U, et al. Increasing resistance against antibiotics in bacteria isolated from the lower urinary tract of an outpatient population of spinal cord injury patients. Urol Int 2004;73:143-8.

12 Bakke A, Digranes A. Bacteriuria in patients treated with clean intermittent catheterization. Scand I Infect Dis 1991;23:577-82.

13 Hill TC, Baverstock R, Carlson KV, et al. Best practices for the treatment and prevention of urinary tract infection in the spinal cord injured population: The Alberta context. Can Urol Association I 2013;7:122-30.

14 Warren JW, Tenney JH, Hoopes JM, et al. A prospective microbiologic study of bacteriuria in patients with chronic indwelling urethral catheters. I Infect Dis 1982;146:719-23.
15 Waites KB, Canupp KC, DeVivo MJ. Epidemiology and risk factors for urinary tract infection following spinal cord injury. Arch Phys Med Rehabil 1993;74: $691-5$.

16 Nicolle LE, Bradley S, Colgan R, et al. Infectious Diseases Society of America guidelines for the diagnosis and treatment of asymptomatic bacteriuria in adults. Clin Infect Dis 2005; 40:643-54

17 Grabe M, Bjerklund-Johansen TE, Botto H, et al. Guidelines on Urological Infections: European Association of Urology; 2012. [cited 2014 6/11/2014]. http:// www.uroweb.org/gls/pdf/17_Urological infections_LR II.pdf

$18 D^{\prime}$ Hondt $F$, Everaert $K$. Urinary tract infections in patients with spinal cord injuries. Curr Infect Dis Rep 2011;13:544-51.

19 Mitchell B, Ware C, McGregor A, et al. ASID (HICSIG)/AICA position statement: preventing catheter-associated urinary tract infections in patients. Healthc Infect 2011;16:45-52.

20 Waites KB, Canupp KC, DeVivo MJ. Eradication of urinary tract infection following spinal cord injury. Paraplegia 1993;31:645-52.

21 Nicolle LE. Urinary tract infections in special populations: diabetes, renal transplant, HIV infection, and spinal cord injury. Infect Dis Clin North Am 2014;28:91-104.

22 Steward DK, Wood GL, Cohen RL, et al. Failure of the urinalysis and quantitative urine culture in diagnosing symptomatic urinary tract infections in patients with long-term urinary catheters. Am J Infect Control 1985;13:154-60.

23 Tambyah PA, Maki DG. The relationship between pyuria and infection in patients with indwelling urinary catheters: a prospective study of 761 patients. Arch Intern Med 2000;160:673-7.

24 NIDDR. The prevention and management of urinary tract infections among people with spinal cord injuries. National Institute on Disability and Rehabilitation Research Consensus Statement. J Am Paraplegia Soc 1992;15:194-204.

25 Hoffman JM, Wadhwani R, Kelly E, et al. Nitrite and leukocyte dipstick testing for urinary tract infection in individuals with spinal cord injury. I Spinal Cord Med 2004;27:128-32.

26 Nicolle LE. Urinary tract infections in patients with spinal injuries. Curr Infect Dis Rep 2014; 16:390

27 Jarvis TR, Chan L, Gottlieb T. Assessment and management of lower urinary tract infection in adults. Aust Prescriber 2014;37:7-9.

28 Kennedy K, Collignon P. Colonisation with Escherichia coli resistant to "critically important" antibiotics: a high risk for international travellers. Eur J Clin Microbiol Infect Dis 2010;29:1501-6.

29 Hooton TM, Bradley SF, Cardenas DD, et al. Diagnosis, prevention, and treatment of catheter-associated urinary tract infection in adults: 2009 International Clinical Practice Guidelines from the Infectious Diseases Society of America. Clin Infect Dis 2010;50:625-63.

30 Lee BS, Bhuta T, Simpson JM, et al. Methenamine hippurate for preventing urinary tract infections. Cochrane Database Syst Rev 2012;10:Cd003265.

31 Edokpolo LU, Stavris KB, Foster HE Jr. Intermittent catheterization and recurrent urinary tract infection in spinal cord injury. Top Spinal Cord Inj Rehabil 2012;18:187-92.

32 Moore KN, Fader M, Getliffe K. Long-term bladder management by intermittent catheterisation in adults and children. Cochrane Database Syst Rev 2007;4: Cd006008.

33 Li L, Ye W, Ruan $\mathrm{H}$, et al. Impact of hydrophilic catheters on urinary tract infections in people with spinal cord injury: systematic review and meta-analysis of randomized controlled trials. Arch Phys Med Rehabil 2013;94:782-7.

34 Consortium for Spinal Cord Medicine. Bladder management for adults with spinal cord injury: a clinical practice guideline for health-care providers. I Spinal Cord Med 2006;29:527-73

35 Eckford SD, Keane DP, Lamond E, et al. Hydration monitoring in the prevention of recurrent idiopathic urinary tract infections in pre-menopausal women. $\mathrm{Br} J \mathrm{Urol}$ 1995;76:90-3.

36 Manz F, Wentz A. The importance of good hydration for the prevention of chronic diseases. Nutr Rev 2005;63(6 Pt 2):S2-5.

37 Salomon J, Denys P, Merle C, et al. Prevention of urinary tract infection in spinal cord-injured patients: safety and efficacy of a weekly oral cyclic antibiotic (WOCA) programme with a 2 year follow-up -an observational prospective study. J Antimicrob Chemother 2006;57:784-8.

38 Mays $\mathrm{R}$, McIntyre A, Mehta $\mathrm{S}$, et al. A review of educational programs to reduce UTIs among individuals with SCI. Rehabil Nurs 2014;39:240-9.

39 Barber DB, Woodard FL, Rogers SJ, et al. The efficacy of nursing education as an intervention in the treatment of recurrent urinary tract infections in individuals with spinal cord injury. SCI Nurs 1999;16:54-6. 